Pediat.Res. 4: 493-499 (1970)

Dimethyl sulfoxide

DNase II

lysosomal membrane neutrophil

progressive septic

granulomatosis

\title{
Zur Ätiologie der progressiven septischen Granulomatose
}

\author{
G. ESGHENBACH \\ Universitäts-Kinderklinik, Marburg a.d.Lahn, Deutschland
}

\begin{abstract}
Extract
Deoxyribonuclease (DNase II) activity in leukocytes of children with progressive septic granulomatosis (PSG) was investigated in blood film preparations by a cytochemical method. Compared with a control group of normal persons with bacterial and viral diseases $(\mathrm{N}=10)$, decreased activity was found in the neutrophil granulocytes of patients with PSG $(\mathrm{N}=5)$. Mean value of the DNase II index of neutrophil granulocytes of patients with PSG was 0.78, that of the control group was 2.93. The activity of the eosinophil granulocytes, lymphocytes, and monocytes corresponded to that of the control group. After heat alteration of the lysosome membranes in the neutrophil granulocytes (heating. of fixated film preparations to $60^{\circ}$ for $60 \mathrm{~min}$ ), and addition of dimethyl sulfoxide to the incubation solution $(0.45 \%, \mathrm{v} / \mathrm{v})$, the DNase II activity in the neutrophil granulocytes of patients with PSG $(\mathrm{N}=4)$ was in the same concentration as the activity found in the granulocytes of the control group $(\mathrm{N}=10)$. In patients with PSG, disordered fusion of lysosomes to phagosomes may be seen as a functional disorder of the lysosome membrane. The absence of stimulation of the hexose-monophosphate transfer of the neutrophil granulocytes of patients with PSG is considered to be a consequence of the fusion disorder of the lysosomes.
\end{abstract}

\section{Spekulationen}

Eigene, bisher nicht abgeschlossene Untersuchungen zeigen, daß in In-vitro-Phagocytoseversuchen durch Zusatz von Dimethylsulfoxid eine starke Zunahme der Bildung der Phagolysosomen der neutrophilen Granulocyten der PSG-Patienten erreicht wird. Wahrscheinlich ergibt sich durch die Gabe von lysosomenmembran-labilisierenden Substanzen eine Möglichkeit, den angeborenen Defekt der neutrophilen Granulocyten auszugleichen und damit eine spezifische Therapie der PSG-Patienten.

\section{Einleitung}

In der Gruppe von Krankheiten insuffizienter Infektabwehr läßt sich die progressive septische Granulomatose (PSG) [7, 33] durch die Untersuchungsergebnisse von HoLmes und Mitarb. [15] als gesonderte Einheit betrachten. Diese Autoren fanden, daß neutrophile
Granulocyten von Patienten mit PSG bestimmte Erreger zwar normal phagocytieren, jedoch nicht oder nur insuffizient digestieren. VoN RODEY und Mitarb. [28] wurde ein entsprechendes Verhalten der Monocyten von PSG-Patienten beschrieben. Die Ursache dieser Digestionsinsuffizienz ist bisher nicht geklärt. Holmes und Mitarb. [15] diskutieren, daß ein Ausbleiben der 
Fusion der Lysosomen mit den Phagosomen in einem ursächlichen Zusammenhang mit der Digestionsinsuffizienz steht.

Die im folgenden beschriebenen Untersuchungen machen es wahrscheinlich, daß die Ursache dieses Defektes der neutrophilen Granulocyten in einer Anormalität der Lysosomenmembran zu sehen ist.

\section{Methodische Angaben und Untersuchungsmaterial}

Durch das von Aronson und Mitarb. [3] und VorBRODT [32] angegebene und von EsaHENBACH [9] für die Anwendung auf Blutzellen modifizierte cytochemische Nachweisverfahren wurde die lysosomale Aktivität der sauren Desoxyribonuclease (DNase II) der Leukocyten in Blutausstrichpräparaten untersucht (vgl. [4]).

Das Prinzip des Nachweises beruht darauf, daß das Substrat hochmolekulare DNS der Inkubationslösung durch die DNase II der untersuchten Zellen zu Oligound Mononucleotiden depolymerisiert wird. Durch die ebenfalls der Inkubationslösung zugesetzte saure Phosphatase wird das in 3'-Stellung stehende Phosphation freigesetzt und mit der Methode der Schwermetallsalzpräzipitation [12] in Form brauner Niederschläge mikroskopisch sichtbar dargestellt (vgl. [8]). Die Intensität des Reaktionsausfalles wurde in 6 Reaktionsgrade $(0-V)$ unterteilt und damit eine Berechnung des mittlerenDNase-II-Index (score) ermöglicht.

In jedem Ausstrichpräparat wurde die Aktivität von 100 neutrophilen Granulocyten, 100 Lymphocyten, 10 eosinophilen Granulocyten und 10 Monocyten beurteilt. In 2 Ausstrichpräparaten einer Kontrollgruppe gelang es nicht, eine genügende Zahl von eosinophilen Granulocyten zu finden, diese Zellart wurde deswegen in diesen Präparaten nicht gewertet.

Das durch das angegebene Verfahren bestimmte Reaktionsergebnis ist nicht ausschließlich abhängig von der Aktivität, sondern auch von der Nachweisbarkeit des Enzyms, also u.a. von der Permeabilität der dieses Enzym umschließenden Membranen für das Substrat. Um diese evtl. unterschiedliche Permeabilität der lysosomalen Membranen beurteilen zu können, wurden die Ausstrichpräparate der Patienten mit PSG und der Kontrollgruppen unter drei verschiedenen Versuchsbedingungen bearbeitet.

1. Durchführung des cytochemischen Verfahrens ohne Modifizierung der Methode,

2. Hitzealteration der lysosomalen Membranen durch Erwärmung der fixierten Ausstrichpräparate während $60 \mathrm{~min}$ auf $60^{\circ}$ vor Beginn der Inkubation,

3. Steigerung der Permeabilität der lysosomalen Membranen durch Zusatz von Dimethylsulfoxid
(DMSO) zur Inkubationslösung in einer Endkonzentration von $0,45 \%(\mathrm{v} / \mathrm{v})$.

Für die Untersuchungen standen Präparate von insgesamt 5 Knaben mit PSG [34] zur Verfügung. Bei diesen Kindern wurde die Diagnose durch den BacteriaKilling-Test [17] oder den Nitroblau-TetrazoliumTest [5] gesichert. Die Aktivitätsbestimmungen mit dem modifizierten Nachweisverfahren waren an Präparaten von lediglich 4 Kindern möglich.

In jeder Untersuchungsgruppe dienten Ausstrichpräparate von 10 Kindern mit bakteriell und viral bedingten Erkrankungen als Vergleich. Es wurden Patienten mit diesen Erkrankungen herangezogen, da nach Zusatz von DMSO zur Inkubationslösung der mittlere DNase-II-Index aller Leukocytenarten dieser Gruppe mit denen dcr PSG-Patientcn übereinstimmt.

\section{Ergebnisse}

Bei hämatologisch gesunden Personen ist die DNase-IIAktivität mit der angegebenen Methode im. Cytoplasma aller Leukocytenarten nachweisbar. Während bakterieller und viraler Erkrankungen ist die Aktivität des Enzyms höher als in infektfreien Perioden. Besonders die neutrophilen Granulocyten lassen diese Abhängigkeit der Aktivität bzw. ihrer Nachweisbarkeit vom Bestehen oder der Ausprägung von entzündlichen Erkrankungen erkennen (vgl. [10]).

Nach Anwendung des nicht modifizierten Nachweisverfahrens zeigen elektiv die neutrophilen Granulocyten der PSG-Patienten im Vergleich zu denen hämatologisch gesunder Kontrollpersonen stark erniedrigte Werte des DNase-II-Index. In eosinophilen Granulocyten, Lymphocyten und Monocyten der PSG-Patienten entspricht die DNase-II-Aktivität der hämatologisch gesunder Kontrollpersonen (Abb.1 und Tab.IA).

Nach Hitzealteration unterschied sich der DNase-IIIndex der neutrophilen Granulocyten der PSG-Patienten nicht von dem entsprechender Zellen hämatologisch gesunder Personen während viraler und bakterieller Erkrankungen.

Durch DMSO-Zusatz zur Inkubationslösung war der DNase-II-Index aller Leukocytenarten - speziell der neutrophilen Granulocyten - höher als nach Anwendung des nicht modifizierten Nachweisverfahrens. Die neutrophilen Granulocyten der PSG-Patienten zeigten gleich hohe Aktivitäten wie die hämatologisch gesunder Vergleichspersonen (Abb.2 und Tab. IC). Die vorliegenden Ergebnisse lassen erkennen, daß bei der PSG die Reaktionsmöglichkeit der DNase II mit dem Substrat elektiv der neutrophilen Granulocyten im Vergleich zu entsprechenden Zellen hämatologisch gesunder Personen vermindert ist. Durch Anwendung 
Table I. Mean DNase II index of leukocyte types ${ }^{1}$

\begin{tabular}{|c|c|c|c|c|c|c|c|c|c|c|c|c|}
\hline \multirow[t]{2}{*}{ Subjects } & \multicolumn{4}{|c|}{$A$} & \multicolumn{4}{|c|}{$B$} & \multicolumn{4}{|c|}{$C$} \\
\hline & NG & $\mathrm{EG}$ & LC & $\mathrm{MG}$ & NG & EG & LG & MG & NG & EG & LG & MG \\
\hline \multicolumn{13}{|c|}{ Control group } \\
\hline 1 & 2.9 & 3.5 & 3.2 & 2.9 & 3.3 & 3.5 & 3.0 & 3.1 & 3.6 & 3.4 & 2.9 & 3.2 \\
\hline 2 & 2.8 & 3.2 & 3.0 & 3.0 & 2.9 & 2.8 & 2.3 & 3.0 & 3.9 & 3.5 & 3.1 & 3.2 \\
\hline 3 & 3.0 & 3.6 & 2.9 & 3.2 & 3.0 & - & 2.7 & 3.0 & 3.5 & 3.9 & 3.1 & 3.4 \\
\hline 4 & 3.0 & 3.5 & 3.0 & 2.9 & 3.2 & 3.0 & 3.0 & 3.0 & 4.6 & 4.3 & 3.3 & 3.3 \\
\hline 5 & 2.9 & 3.7 & 3.0 & 3.1 & 2.9 & 2.9 & 2.9 & 2.7 & 3.2 & 3.8 & 3.2 & 3.0 \\
\hline 6 & 3.1 & 3.3 & 3.1 & 3.0 & 2.8 & 2.7 & 2.8 & 2.9 & 3.0 & 3.6 & 2.9 & 3.0 \\
\hline 7 & 3.0 & 3.4 & 3.0 & 3.0 & 3.0 & 2.8 & 2.8 & 2.9 & 3.9 & 4.0 & 3.0 & 3.3 \\
\hline 8 & 2.6 & 2.9 & 2.4 & 3.0 & 2.4 & - & 2.0 & 2.3 & 3.8 & 4.0 & 3.0 & 3.5 \\
\hline 9 & 3.1 & 3.0 & 2.9 & 3.0 & 3.1 & 3.0 & 2.8 & 2.8 & 3.6 & 3.8 & 3.0 & 3.1 \\
\hline 10 & 2.9 & 2.9 & 2.5 & 2.8 & 2.9 & 3.0 & 3.0 & 3.0 & 3.0 & 3.6 & 2.9 & 2.9 \\
\hline Mean & 2.93 & 3.30 & 2.90 & 2.99 & 2.95 & 2.96 & 2.73 & 2.86 & 3.61 & 3.79 & 3.04 & 3.19 \\
\hline SD & 0.15 & 0.29 & 0.25 & 0.11 & 0.25 & 0.24 & 0.33 & 0.23 & 0.48 & 0.27 & 0.19 & 0.19 \\
\hline \multicolumn{13}{|c|}{ Patients with PSG } \\
\hline$G B$ & 1.3 & 3.4 & 2.9 & 3.0 & 2.9 & 2.8 & 2.9 & 3.0 & 3.8 & 3.2 & 3.1 & 3.2 \\
\hline$W F$ & 1.4 & 3.4 & 2.4 & 2.9 & & & & & & & & \\
\hline$K S$ & 0.4 & 3.5 & 3.0 & 2.9 & 2.9 & 3.0 & 2.8 & 2.9 & 3.3 & 3.3 & 2.7 & 3.1 \\
\hline$M W$ & 0.1 & 3.0 & 2.8 & 2.8 & 3.0 & 3.0 & 2.9 & 3.0 & 4.0 & 4.4 & 2.9 & 3.2 \\
\hline$B V \mathcal{F}$ & 0.7 & 3.4 & 2.7 & 2.5 & 2.5 & 3.0 & 2.7 & 2.5 & 3.2 & 3.5 & 2.8 & 2.8 \\
\hline
\end{tabular}

${ }^{1}$ Activity of neutrophil granulocytes (NG), eosinophil granulocytes (EG), lymphocytes (LC), and monocytes (MC) of normal persons with bacterial or viral inflammations (control group) and of children with progressive septic granulomatosis (PSG) after applying cytochemical method without modification of method $(A)$, after heat alteration of preparations $(B)$, and after adding dimethyl sulfoxide to incubation solution $(C)$.

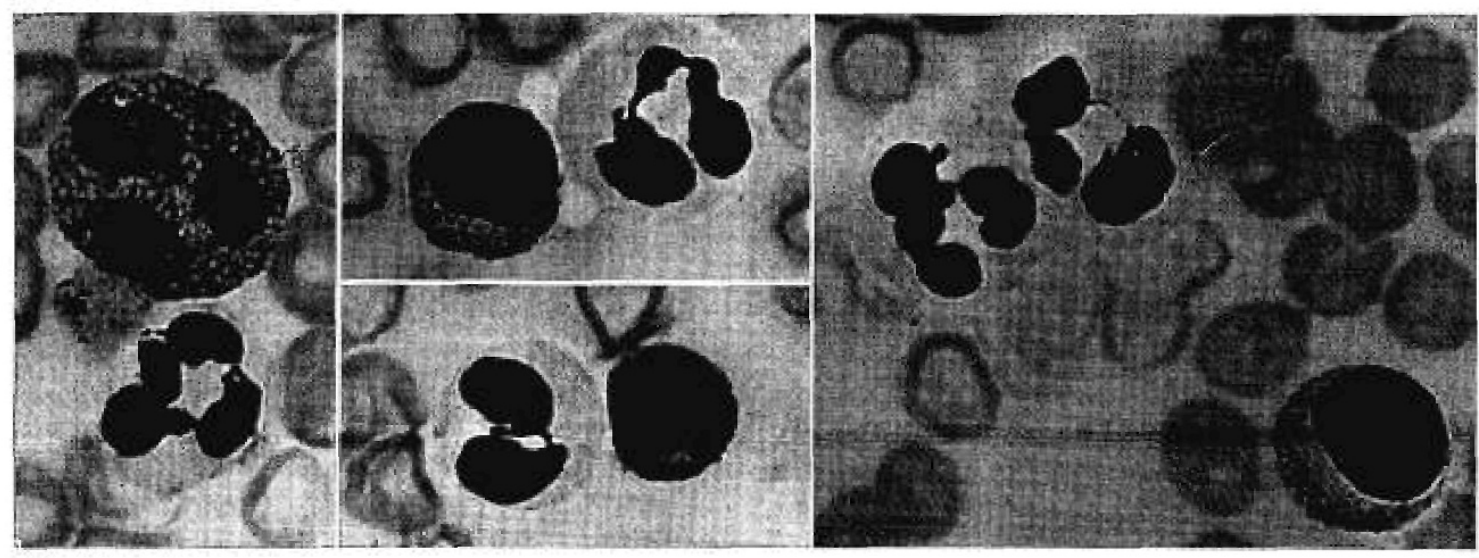

Fig. 1. DNase II activity of leukocytes in a child with progressive septic granulomatosis after applying identification method without modification. The neutrophil granulocytes show little or no enzyme activity, the lymphocytes and one eosinophil granulocyte, however, show high activities. $\times 2,000$. 


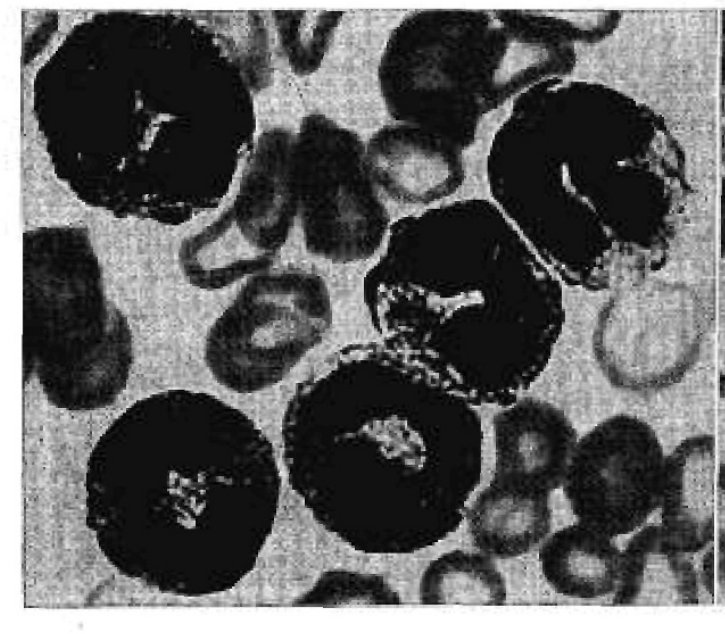

Fig. 2. DNase II activity of neutrophil granulocytes and of one lymphocyte in a child with progressive septic granulomatosis after adding dimethyl sulfoxide to the

von zwei Modifikationen des Verfahrens gelingt es, die Aktivität des Enzyms in diesen Zellen in normaler Intensität nachzuweisen. Erwärmung der Ausstrichpräparate und Zusatz von DMSO zur Inkubationslösung bewirken eine «Demaskierung » und ermöglichen den cytochemischen Nachweis des lysosomalen Enzyms DNase II in neutrophilen Granulocyten von PSG-Patienten.

Die DNase-II-Aktivität der Monocyten - bzw. ihre Nachweisbarkeit in diesen Zellen - von PSG-Patienten wich nicht $a b$ von der hämatologisch gesunder Personen.

\section{Diskussion}

Richtungweisend für die Ätiologie der PSG waren die bereits erwähnten Untersuchungsergebnisse von Holmes und Mitarb. $[15,16]$ sowie von QuIE und Mitarb. [26], daß die Leukocyten der PSG-Patienten bestimmte Bakterien nach der Phagocytose nicht bzw. in unphysiologisch geringer Intensität digestieren. Diese Befunde wurden bestätigt durch KAPLAN und Mitarb. [20], ANDERsen und Mitarb. [2], Thompson und Mitarb. [31], Hitzig und Mitarb. [18]. Von gleicher Bedeutung ist die Erkenntnis, daß die neutrophilen Granulocyten von PSG-Patienten nach stattgehabter Phagocytose die Degranulation und die Entstehung von cytoplasmatischen Vakuolen vermissen lassen [26, $17,2]$. Beide Phänomene finden ihre Erklärung in einer Störung der Fusion von Lysosomen und Phagosomen. Holmes und Mitarb. [17] diskutieren, daß die Lysosomen nicht ruptuieren und ihren Inhalt nicht in die Phagosomen abgeben können (vgl. [21]).

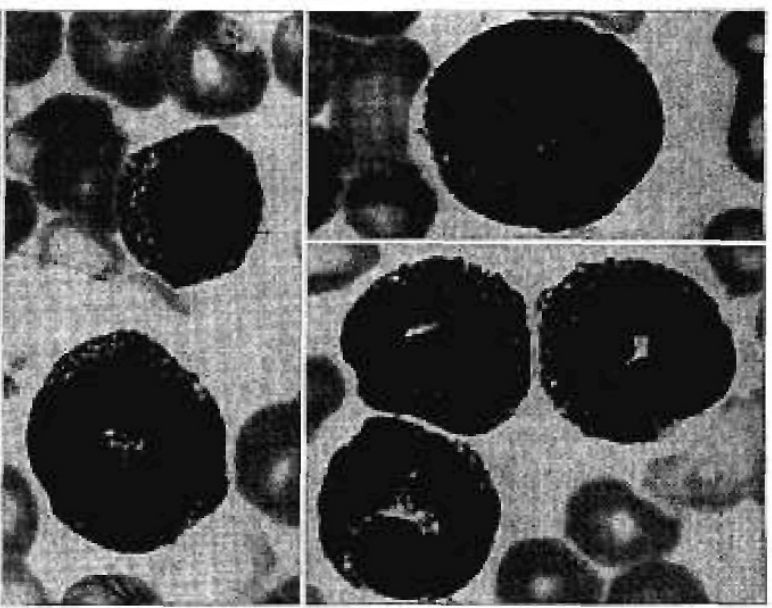

incubation solution (final concentration $0.45 \%$ ). The neutrophil granulocytes show high enzyme activity. $\times 2,000$.

Der negative Ausfall des NitroblautetrazoliumTestes wurde von BAEHNER und Mitarb. [5] als Mangel an NADH-Oxidase-Aktivität gedeutet (vgl. auch [18] und [27]). Holmes und Mitarb. [17] fanden in Leukocyten von PSG-Patienten jedoch normale Aktivitäten der NADH- und NADPH-Oxidasen. BAEHNER und Mitarb. [5] sehen in der mangelnden Stimulation der NADH-Oxidase-Aktivität der PSG-Leukocyten die Ursache der Erkrankung. Meines Wissens liegen im Schrifttum keine Angaben darüber vor, ob die Stimulation der NADH-Oxidase-Aktivität von der Ingestion, zu der die PSG-Leukocyten normal befähigt sind, oder von der Fusion der Lysosomen mit den Phagosomen, zu der die Granulocyten der PSG-Patienten nicht in der Lage sind, abhängig ist. Im letzteren Falle wäre also eine Stimulation der NADH-Oxidase-Aktivität der PSG-Granulocyten nicht zu erwarten.

Leukocyten hämatologisch normaler Personen reagieren nach der Ingestion zellfremden Materials mit einer Stimulation des Hexose-Monophosphat-Weges, aus dem die für die Digestion erforderliche Energie zur Verfügung gestellt wird. Diese Regulation erfolgt über NADP [17]. PSG-Leukocyten dagegen lassen die Reaktion dieses Stoffwechselweges nach stattgehabter Ingestion vermissen [17]. HoLmes und Mitarb. [17] zeigten, daß der Hexose-Monophosphat-Weg der PSG-Leukocyten stimulierbar ist, daßdiese Zellen über normale NADPH-Konzentrationen verfügen und in Homogenaten der PSG-Leukocyten normale Aktivitäten der NADPH-Oxidasen nachweisbar sind. Dadurch ist erwiesen, daß die biochemischen Voraussetzungen dieses Stoffwechselweges in Leukocyten von PSG-Patienten gegeben sind. Das Ausbleiben der Re- 
aktion des Hexose-Monophosphat-Weges der PSGLeukocyten nach der Ingestion ist wahrscheinlich auf eine mangelnde Stimulation zurückzuführen.

In PSG-Leukocyten wurden normale Aktivitäten der sauren Phosphatase und $\beta$-Glucuronidase [14] sowie Lysozym und Phagocytin [26] nachgewiesen. Untersuchungen über die enzymatische Gesamtausstattung der Lysosomen dieser Zellen, die Bindung der Enzyme an Membranen und über die Freisetzung der Enzyme liegen bisher im Schrifttum nicht vor.

Die eigenen Untersuchungen stellen einen Beitrag zur Klärung der Fusion der Lysosomen mit den Phagosomen dar: Es wird gezeigt, daß eine Anormalität der Lysosomenmembranen elektiv der neutrophilen Granulocyten bei der PSG vorliegt. Durch das angegebene cytochemische Verfahren ist das lysosomale Enzym DNase II in den neutrophilen Granulocyten der PSGPatienten nicht oder im Vergleich zu hämatologisch gesunden Kontrollpersonen stark vermindert nachweisbar. Nach Hitzealterierung der Leukocyten im fixierten Ausstrichpräparat - DNase II ist ein hitzestabiles Enzym [30] - oder noch wirkungsvoller nach Steigerung der Permeabilität der Lysosomenmenbranen durch Zusatz von Dimethylsulfoxid zur Inkubationslösung ist das Enzym in den neutrophilen Granulocyten der Patienten mit einer Aktivität nachweisbar, die der der Kontrollpersonen entspricht. Die eosinophilen Granulocyten, Lymphocyten und Monocyten der PSG-Patienten lassen dagegen keine Abweichung der Nachweisbarkeit des Enzyms von entsprechenden Zellen der Normalpersonen erkennen. Die Diskrepanz zwischen normaler Nachweisbarkeit der DNase II und der von RODEY und Mitarb. [28] gezeigten verminderten bactericiden Aktivität der Monocyten kann bisher nicht erklärt werden. Zur Zeit wird die Kapazität der PSG-Monocyten, im Phagocytosetest Vakuolen zu bilden, untersucht.

Misch und Mitarb. [23] zeigten an Lysosomensuspensionen aus Rattenleber, daß durch Zusatz von DMSO die Nachweisbarkeit der sauren Phosphatase durch Steigerung der Permeabilität der Lysosomenmembranen für das Substrat (Na- $\beta$-Glycerophosphat) gesteigert wird. Dieser Effekt des DMSO wird als Demaskierung bezeichnet. Von GANDER und Mitarb. [11] wurde dieser Befund bestätigt. Ebenfalls Axtrand und Mitarb. [1] beschrieben eine Steigerung der Permeabilität von Organellenmenbranen durch DMSO.

Die Ergebnisse der vorliegenden Untersuchungen lassen die Schlußfolgerung $\mathrm{zu}$, daß mit Hilfe des angegebenen cytochemischen Verfahrens in neutrophilen Granulocyten von PSG-Patienten das lysosomale Enzym DNase II erst durch Anwendung permeationsfördernder Faktoren in einer Intensität, die der hämatologisch normaler Personen entspricht, nachweisbar wird. Die Permeabilität der lysosomalen Membranen der neutrophilen Granulocyten dieser Patienten ist also offenbar im Vergleich zu der der Kontrollgruppe vermindert.

Das Ausbleiben der Vakuolenbildung der neutrophilen Granulocyten der PSG-Patienten nach Phagocytose sprechen für eine Störung der Fusion von Lysosomen und Phagosomen, für die die Ruptur der Lysosomenmembran notwendige Voraussetzung ist. Die eigenen Ergebnisse in Verbindung mit den referierten Befunden machen es wahrscheinlich, daß die Stabilität der Lysosomenmembran elektiv der neutrophilen Granulocyten der PSG-Patienten unphysiologisch hoch ist. Es ist anzunehmen, daß dadurch die Unfähigkeit der Lysosomenmembran zu rupturieren und somit die Insuffizienz der Digestion phagocytierten Materials bedingt ist. Das Ausbleiben der Stimulation des Hexose-Monophosphat-Weges trotz normaler Phagocytose wäre dann als sekundäres Phänomen aufzufassen.

\section{Zusammenfassung}

In Leukocyten von Patienten mit progressiver septischer Granulomatose (PSG) wurde durch ein cytochemisches Verfahren die Aktivität der lysosomalen Desoxyribonuclease untersucht. Elektiv die neutrophilen Granulocyten zeigten eine starke Verminderung der Aktivität. Nach Hitzealterierung und Anwendung einer die Permeabilität steigernden Substanz (Dimethylsulfoxid) ist das Enzym mit einer Aktivität nachzuweisen, die der hämatologisch gesunder Vergleichspersonen entspricht. Diese Befunde in Verbindung mit den bei der PSG bekannten Phänomenen fehlender Vakuolenbildung und Degranulation im Phagocytose-Test als Ausdruck einer mangelnden Phagolysosomenentstehung machen es wahrscheinlich, daß die Ursache dieser Erkrankung in einer Funktionsstörung der Lysosomenmenbran zu sehen ist.

\section{Summary}

The activity of lysosomal deoxyribonuclease was studied by cytochemical means in leukocytes of patients suffering from progressive septic granulomatosis (PSG). Only the neutrophil granulocytes revealed a considerable decrease in enzymatic activity. When the cells were exposed to heat or dimethylsulfoxide, a substance found to increase membrane permeability, deoxyribonuclease activity was equal to that of normal controls. Our findings suggest that the cause of this disease is probably a functional deficiency of the lysosomal membrane. 


\section{Literatur und Noten}

1. Altuand, P.D.; Highman, B.; Nelson, B.D. and Garbus, J.: Effects of dimethyl sulfoxide on serum enzymes and tissues in exercised rats. Life Sci. 5: 375 (1966).

2. Andersen, V.; Koch, C.; Vejlsgaard, R. and Wilken-Jensen, K. : Fatal granulomatous disease. Acta paediat., Uppsala 57: 110 (1968).

3. Aronson, J.; Hempelmann, H. L. and Okada, S. Preliminary studies on the histological demonstration of desoxyribonuclease II by adaptation of the Gomori acid phosphatase method. J. Histochem. Cytochem. 6: 255 (1958).

4. Atwal, O.S.; Frye, F.L. and Enright, J.B.: Acid deoxyribonuclease activity in peripheral leucocytes in swiss mice. Nature, Lond. 205: 185 (1965).

5. Baehner, R.L. and Nathan, D.G.: Leukocyte oxydase: Defective activity in chronic granulomatous disease. Science 155: 835 (1967).

6. Berendes, H.; Bridges, R.A. and Good, R.A.: A fatal granulomatosis of childhood: The clinical study of a new syndrome. Minn. Med. 40: 309 (1957).

7. Carson, M. L.; Ghadwick, D.L.; Brubaker, C. A.; Cleland, R.S. and Landing, B.H.: Thirteen boys with progressive septic granulomatosis. Pediatrics 35: 405 (1965).

8. Eschenbach, C.: Zytochemischer Nachweis der Nukleasen-Aktivität in Leukosezellen; in: STACHER: Chemo- und Immunotherapie der Leukosen und malignen Lymphome (Bohmann-Verlag, Wien 1969).

9. Eschenbach, G.: Cytochemischer Nachweis von saurer Desoxyribonuclease im Cytoplasma von Blutzellen. I. Methode. Histochemie 24: 85 (1970).

10. Eschenbach, C.: Cytochemischer Nachweis von saurer Desoxyribonuclease im Cytoplasma von Blutzellen. II.Aktivität der sauren Desoxyribonuclease im Cytoplasma von Leukocyten während akuter Infektionen. Hisochemie, in Vorbereitung.

11. Gander, E.S. and Moppert, J. M. : Der Einfluß von Dimethylsulfoxid auf die Permeabilität der Lysosomenmembran bei quantitativer und qualitativer Darstellung der sauren Phosphatase. Histochemie 20: 211 (1969).

12. Gomori, G.: Histochemical methods for acid phosphatase. J. Histochem. Cytochem. 4: 453 (1956).

13. Good, R.A.; Quie, P.G.; Windhorst, D.B.; Page, A.R.; Rodey, G.E.; White, J.; Wolfson, J.J. and Holmes, B.H.: Fatal (chronic) granulomatous disease of childhood: a hereditary defect of leukocyte function. Sem. hematol. 5: 215 (1968).

14. Holmes, B.: Studies on the metabolic activity of leukocytes from patients with a genetic abnormality of phagocytic function. (Ph.D. thesis, University of Minnesota, Minneapolis 1966.)

15. Holmes, B.; Qure, P.G.; Windhorst, D.B. and Good, R. A. : Fatal granulomatous disease of childhood: an inborn abnormality of phagocytic function. Lancet $i$ : 1225 (1966).

16. Holmes, B.; Quie, P.G.; Windhorst, D.P.; PolLARA, B. and Good, R.A.: Protection of phagocytized bacteria from the killing action of antibiotics. Nature, Lond. 210: 1131 (1966).

17. Holmes, B.; PAGE, A. R. and Good, R. A.: Studies of the metabolic activity of leukocytes from patients with a genetic abnormality of phagocytic function. J.clin. Invest. 46: 1422 (1967).

18. Hrtzig, W.H.; Molz, G.; Plüss, H.J. and ReNNER, R.: Progressive septische Granulomatose. Helv. paediat. Acta 24: 246 (1969).

19. Johnston, R.B. and MaMurry, J.S.: Chronic familial granulomatosis. Pediatrics 40: 808 (1967).

20. Kaplan, E. L. : zit. nach 13.

21. Kaplan, E. L.; Laxdal, T. and Quie, P. G.: Studies of polymorphonuclear leukocytes from patients with chronic granulomatous disease of childhood bactericidal capacity for streptococci. Pediatrics 41: 591 (1968).

22. MagFarlane, P.S.; Speirs, A.L. and SommerVILLE, R. G. : Fatal granulomatous disease of childhood and benign lymphocytic in filtration of the skin (congenital dysphagocytosis). Lancet $i: 408$ (1967).

23. Mrsch, D. W. and Misch, M. S. : Reversible activation of lysosomes by dimethyl sulphoxide. Nature, Lond. 221: 862 (1969).

24. NeIDHARDT, M.: Die progressive septische Granulomatose. Herbsttagung der Rheinisch-Westfälischen Kinderärztevereinigung, Duisburg, 15. Nov. 1969.

25. Quie, P. G.; KaPlan, E. L.; PAge, A. R.; Gruskay, F.L. and Malawista, S.E.: Defective polymorphonuclear-leukocyte function and chronic granulomatous disease in two female children. New Engl.J. Med. 278: 976 (1968).

26. Quie, P. G.; White, J. G.; Holmes, B. and Good, R.A.: In vitro bactericidal capacity of human polymorphonuclear leukocytes: diminished activity in chronic granulomatous disease of childhood. $\mathrm{J}$. clin. Invest. 46: 668 (1967).

27. Renner, R. and Hrtzig, W.H.: Die septische Granulomatose: eine $\mathrm{x}$-chromosomal vererbte Funktionsstörung der Granulocyten. Schweiz. med. Wschr. 98: 1673 (1968).

28. Rodey, G.E.; Park, B.H.; Windhorst, D. B. and Good, R.A.: Defective bactericidal activity of monocytes in fatal granulomatous disease. Blood 33: 813 (1969). 
29. Rosenmayr, F. and Weippt, G.: Familiäre septische Granulomatose. Wien.klin. Wschr. 81: 635 (1969).

30. Stebert, G.: Desoxyribonucleasen; in: LANG und LEHNARTZ: Handbuch der physiologisch- und pathologisch-chemischen Analysen, 6. Bd. Teil B, 10. Aufl. (Springer, Berlin 1966).

31. Thompson, E.N.; Chandra, R.K.; Cope, W.A. and Soothill, J.F.: Leukocyte abnormality in both parents of a patient with chronic granulomatous disease. Lancet $i$ : 799 (1969).

32. Vorkrodt, A.: Histochemical studies on the intracellular localization of acid deoxyribonuclease. J. Histochem. Cytochem. 9: 647 (1961).

33. Synonyme: fatal granulomatous disease of childhood $[6,13]$; chronic granulomatous disease [25, 26]; chronic familial granulomatosis [19]; congenital dysphagocytosis [22].
34. G.B.: veröffentlicht durch Rosenmayr und Mitarb. [29] und Hitzig und Mitarb. [18]; W. F.: veröffentlicht durch Hitzig und Mitarb. [18]; K.S. und $M . W .:$ veröffentlicht durch NEIDHARDT [24]; B.V.F.: bisher nicht veröffentlicht.

35. Ich danke Herrn Prof.Dr. Baumann (Kinderspital Aarau), Herrn Prof.Dr. Wrlken-Jensen (Rigshospitalet, Boerneallergieklinikken, Kopenhagen), Herrn Doz.Dr. Rosenmayr (Univ.-Kinderklinik Wien) und Herrn Doz.Dr. NeIDhardt (Univ.Kinderklinik Mainz) für die Überlassung der Blutausstrichpräparate der Patienten.

36. Die Untersuchungen wurden mit Sachbeihilfen der Deutschen Forschungsgemeinschaft durchgeführt.

37. Requests for reprints should be addressed to: Dr. G. Eschenbach, Universitäts-Kinderklinik, D-355 Marburg a.d. Lahn (BRD).

38. Accepted for publication June 10, 1970. 